\title{
Application of a chamfered mold to improve corner defects of HSLA during slab continuous casting
}

\author{
P. Hu ${ }^{1,2}$, H. Zhang ${ }^{1}$, M. Wang ${ }^{1}$, M. Zhu ${ }^{2}$, X. Zhang ${ }^{1}$, Y. Zhang ${ }^{2}$ \\ and Z. Zhang ${ }^{2}$ \\ 1 National Engineering and Research Center for Continuous Casting Technology, Center Iron \\ and Steel Institute, Beijing 100081, P.R. China \\ e-mail: hugkj-545@163.com \\ 2 School of Materials and Metallurgy, Northeastern University, Shenyang 110819, P.R. China
}

Key words:

Continuous casting; HSLA slab; chamfered mold; transverse crack; hot transporting ratio

Received 3 July 2014

Accepted 12 January 2015

\begin{abstract}
Through the study of the slab continuous casting production process and laboratory simulation, the mechanism of transverse corner cracks on a micro-alloyed high-strength steel slab was explored. Then, a chamfered mold was developed and tested on the caster. The results show that on one hand, the chamfered mold could raise the temperature of the corner in the mold, and on the other hand, the higher temperature of the corner of the slab was an effective way to decrease the corner stress at the straightening point. The straightening deformation rate on the chamfered slab was lower than that on the square slab. Using the large chamfer mold technology, the optimization design of the foot roll structure and second cooling, the transverse corner crack was improved effectively. The hot transporting ratio was increased without the cutting angle.
\end{abstract}

I $\mathrm{n}$ the production of micro-alloyed steel, surface quality defects of the slab and plate have been a concern for the steel industry worldwide. For many years, scientists at home and abroad have created many of theories and application of the results concerning the formation mechanism of slab transverse cracks. However, this technical problem has not been solved so far, despite being studied by ThyssenKrupp, VIA, Nippon Steel and Posco Steel. Chamfered mold technology is recognized as the world's best solution to solve this problem completely, but its application is very difficult. Since the technology was proposed in the 1980s, there was no precedent for its successful application. There were three key technical problems to be resolved. The first was the insufficient cooling of a copper mold with a narrow chamfered corner [1-5]. The second was a limit on the cast speed. The third was a limit on the lifetime.

This project studied typical niobium, vanadium, titanium micro-alloyed steels on a slab caster in Hanbao steelworks of the Handan Iron and Steel Company. The surface crack defect formation mechanism was studied by basic research in the laboratory, computer simulation and industrial tests. The set technology of the chamfered slab mold, long-life technology of the chamfered mold, the whole process efficiency of the technology of the casting-rolling interface, precisely controlled technology of the chamfered slab cooling system, technology of the caster roll gap and outside the arc rapid adjustment were developed and they were implemented successfully in a largescale industrial production process.

The technology roadmap of the project is shown as follows: Site investigation $\rightarrow \mathrm{Ba}-$ sic research in the laboratory $\rightarrow$ Basic theoretical analysis and process development $\rightarrow$ Application $\rightarrow$ technology optimized and scale applications. The general idea was illustrated as follows:

(1) It was completed firstly by investigate of the corner transverse cracking and rolled steel defects, and a stage of the research on the formation mechanism. The study of the formation mechanism of cracks was completed, on the surface 
of the micro-alloy slab in the continuous casting process, by literature research, numerical simulation, field tests, online testing of the slab temperature and thermal simulation in the lab.

(2) The precipitation behavior of niobium carbonitride and titanium nitride was studied using the theory for corner transverse cracks of a micro-alloy steel billet on the outer arc. The quality of the slab corner on the foot roll area, top curved area and straightening zones for different locations was detected and analyzed to explore the mechanism of slab transverse cracking of micro-alloyed steel on the outer arc angle.

(3) A mathematical model of the steel flow, heat transfer and macroscopic solidification within a right-angled and chamfered mold, stress-strain model was established for peritectic, hypoperitectic micro-alloyed steels, as well as a stressstrain model during the straightening process, and the effect of different corner shapes on the molten steel, temperature, solidification process and stress-strain during the straightening process was analyzed. The theory of micro-alloyed slab corner cracks solved by chamfered mold technology was adopted, and an optimized design for the face shape of the narrow copper of the chamfered mold was developed.

(4) Numerical simulation of the cooling water flow in the mold's narrow faces was studied, combined with the actual structure of the device, for the study of the solidification shrinkage factor in the mold. The theoretical basis of cooling and taper control in the chamfered mold is shown.

(5) A large chamfer mold for the control of corner transverse defects was developed, and the chamfer mold technology and equipment applied in the caster of Hanbao steelworks. They were a chamfered narrow face copper, chamfered narrow face foot roll and chamfered dummy head pad, and tested in industrial application.

(6) The precise cooling control technology of the chamfered slab was developed by optimization and innovation of a secondary cooling system in the caster. Suitable secondary cooling process parameters were used to reduce surface defects and improve the internal quality of the chamfered slab.

(7) The technology of the caster equipment protection was innovated, which was a quick adjustment of the caster segments roll gap and outside the arc, to ensure efficient and stable production of the chamfered slab.

\section{Experimental device and method}

\subsection{Optimized design and application for the copper narrow face of the chamfered mold}

According to laboratory-based research and numerical simulation results, combined with slab caster working conditions, three patented technologies and related equipment were developed. They are "A chamfered narrow side copper plate for mold with funnel-shaped curved surface" [6], "A chamfered narrow side copper plate with combined cooling structure" [7], "A cooling and fixed structure for the chamfered narrow side copper plate of mold" [8].

\subsubsection{A chamfered narrow side copper plate for a mold with a funnel- shaped curved surface}

A conventional continuous casting mold was used that was tapered so that the upper part was larger and the lower part was smaller. Although the chamfered bevel faces were flat with a variety of curved surfaces, despite the technology of molds with chamfered copper narrow faces already being used in actual production, a chamfered tapered slope was not considered in existing techniques for chamfer molds. Especially, cracks occurred while the beveled chamfer was larger than $30 \mathrm{~mm}$, without the consideration of solidification shrinkage on the chamfered face, and the cooling effect of the chamfered face was poor.

Against the defects and deficiencies of the technology, a chamfered narrow side 
copper plate for a mold with a funnelshaped curved surface was developed in the project. Figure 1 shows the chamfer mold's narrow face copper plate, and Figure 2 shows the curves of the working face, while Figure 3 shows chamfered mold narrow side copper site photos.

\subsubsection{A chamfered narrow side copper plate with a combined cooling structure}

The cooling structure design of the copper corner was rarely involved from the chamfered mold technology and patents obtained. Although incidences of corner transverse cracking were decreased, the effect of the chamfer mold technology was reduced, as corner longitudinal defects of the chamfered slab occurred and even breakout due to insufficient corner cooling. Because of these reasons, a chamfered narrow side copper plate with combined cooling structure was developed in this project. Figure 4 shows a schematic diagram of the combined cooling structure.

\subsubsection{A cooling and fixed structure for the chamfered narrow side copper plate of the mold}

Figure 5 shows a schematic diagram of the chamfered mold's cooling and fixing device structure. The apparatus includes a mold with chamfered narrow surface copper (1) and a backplane tank (4). The cooling tank on the narrow face copper of the chamfered mold was separated into cooling tanks on two sides (6) and a cooling tank in the middle of the central region (7), by the two rows of bolt holes (5). The narrow face copper (1) and backplane tank (4) were connected and fixed by bolts (3), and an annular sealing strip of water (3) was set. The use life was increased and corner transverse cracks were controlled effectively by a cooling and fixed structure for the chamfered narrow side copper plate of the mold mentioned above.

Major breakthroughs were developed in large chamfer mold technology and key equipment by this project. The first was the cooling method by the combination of the

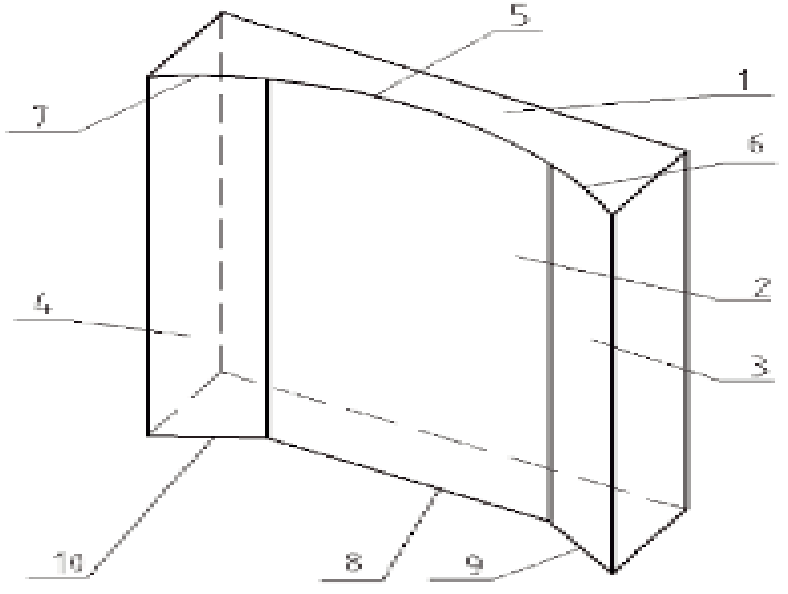

Fig. 1. Chamfer mold's narrow face copper plate.
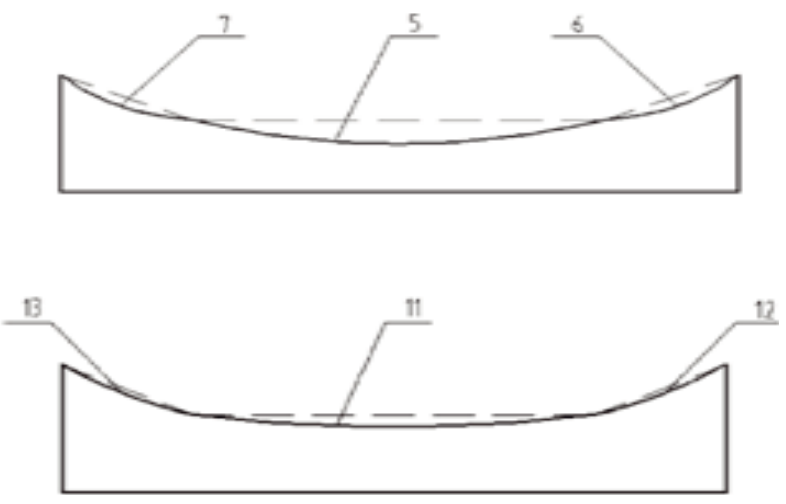

Fig. 2. Curves schematic diagram of the structure of the working face.

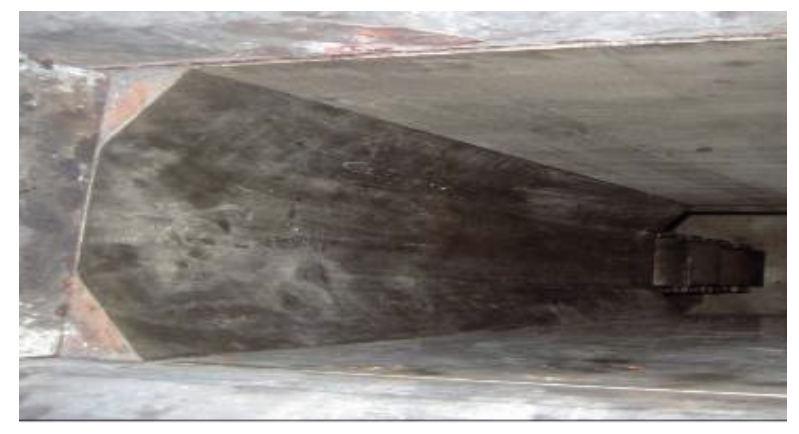

Fig. 3. Chamfered mold narrow side copper site photos.

hole with a water slot, cooling of the corner was strengthened by this structure, and longitudinal breakout was controlled effectively. The second was the work face of the chamfered mold using a funnel-shaped surface, managing the shrinkage characteristics during the solidification process of liquid steel, and heat transfer of the corner was enhanced. The third was the optimized design 


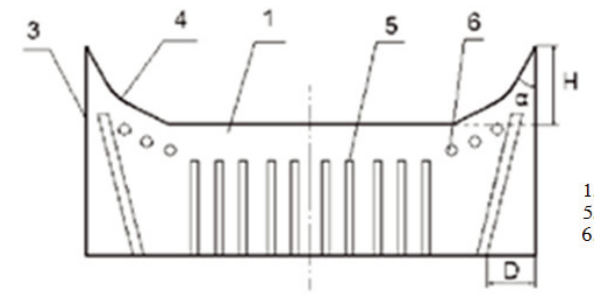

Fig. 4. Schematic diagram of the combined cooling structure.

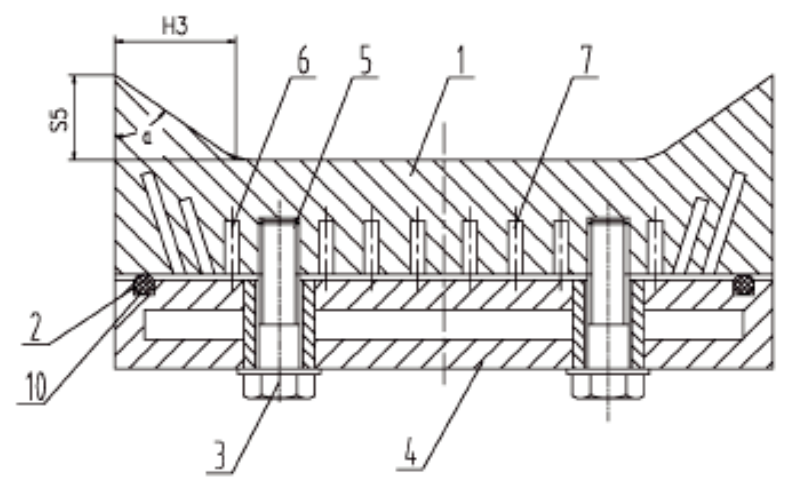

Fig. 5. Schematic diagram of the chamfered mold cooling and fixing device structure.

of the chamfered face angle and the length, and slab bend and force states of straightening were improved while corner temperature was increased effectively.

\subsection{Design and innovation of the chamfering slab supporting foot roll system and long-life technology of the chamfering mold}

\subsubsection{Design and innovation of the chamfering slab supporting foot roll system}

The foot roll should be optimally designed, due to the use of the chamfered narrow face on the mold side and the change in the slab shape. The variety of new systems supported and a suitable foot roll billet for the chamfered mold were designed and developed. They are "A new support roll for the chamfered slab", "A suitable multisection support roll applies for the chamfered slab", and the other two new patents. The slab's narrow face could be supported

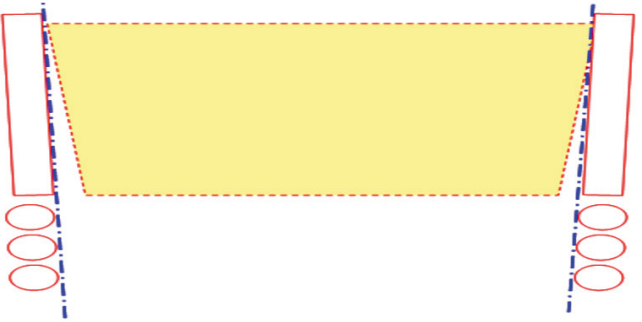

Fig. 6. Schematic diagram of the narrow side foot roll and chamfer plate.

by the chamfered roll. Longitudinal cracks would be avoided, and the use life of the narrow face of the mold would be increased, while high speed would be provided.

\subsubsection{Long-life technology of the chamfered mold}

\subsubsection{Adjusting the center design of the narrow side rolls to the chamfered copper plate}

As shown in Figure 6, the foot rolls on the first row were chamfered foot rolls without a disc spring group buffer on the foot roller's rear. The foot rolls on the second and third rows were cylindrical foot rollers, and the disc spring set was compressed $5 \mathrm{~mm}$, and made the foot rolls on the first, second and third rows on one level flat with the assembly to maintain the accuracy $\pm 0.1 \mathrm{~mm}$. This assembly precision and requirement sufficiently supported the force to support the chamfering slab's side area to avoid bulging and deformation, and this specification was the one key technology of chamfered molds at Handan Iron \& Steel Company to improve their long life.

\subsubsection{Narrow sides' tapered design and application management of the mold}

A tapered structure was adopted in the high direction of the chamfer mold's narrow face copper. The linear offset was $1.2 \mathrm{~mm}$ in the area of $0 \sim 240 \mathrm{~mm}$ from the upper part of the copper plate. Taper adjustment should increase by $0.5 \mathrm{~mm}$ after running of 500 furnaces on-line (Fig. 7) to compensate for wear of the narrow copper surface. Difficulty in forming an air gap between the mold and 


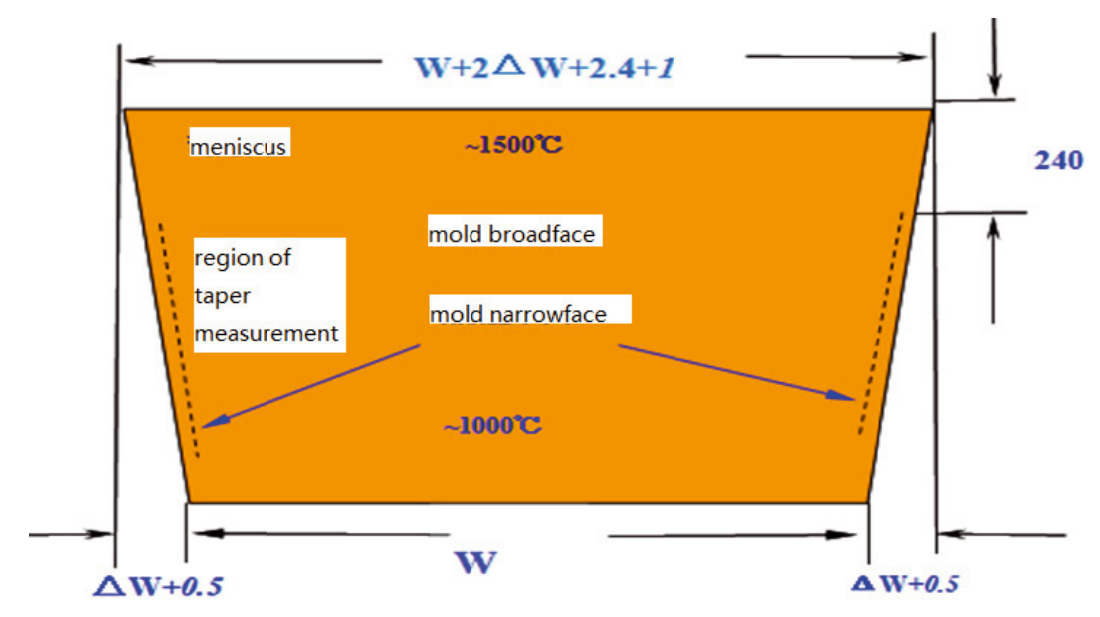

Fig. 7. Schematic diagram of the mold's narrow side tapered design and application.

the copper plate was ensured, with contact made uniformly on the total length of the mold and rapid solidified shell formation. Research and development of the taper change corresponded to specifications in the actual production, and corner longitudinal crack and the corner longitudinal breakout problems were solved effectively. The patents broke the technology's bottlenecks of limitations of the technology industrial application, while ensuring the long-life operation of the chamfer mold.

\subsubsection{The precise control technology of the cooling system for chamfered slabs}

As with conventional rectangular slabs, cooling and solidification of chamfered slabs involves first and secondary cooling zones. The cooling system of the continuous casting machine with chamfered molds in Hanbao Steel was optimized. The precise control technology of the cooling system for chamfered slabs was established. High-speed operation and the production of all steel for chamfered slabs were achieved.

In the first cooling zone, the frame of the narrow side and the cooling water channel of the backboard in the original mold were improved (Figs. 8 and 9). The groove and hole were combined. The slender holes were cleverly reamed. The $\varphi 30$ double hole in the narrow frame was creatively converted into an oblong hole. After the improvement, the

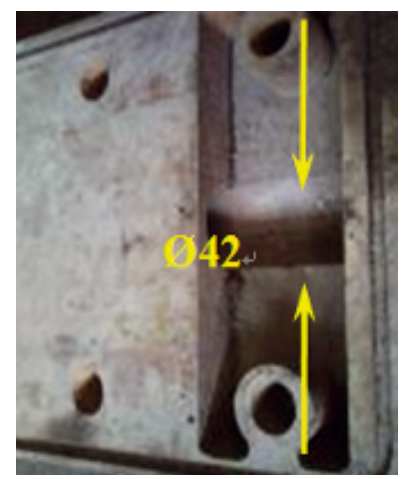

Fig. 8. Waterway before improvement.

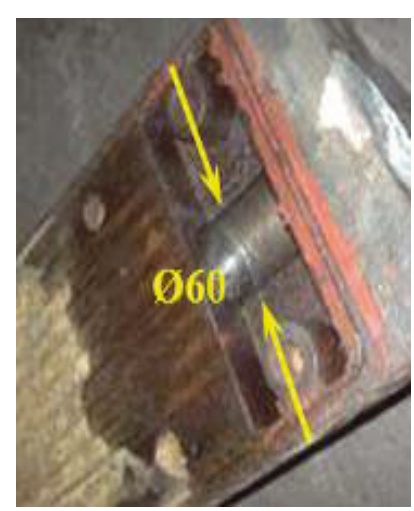

Fig. 9. Improved waterways.

flow of cooling water in the narrow side is not less than $560 \mathrm{~L} / \mathrm{min}$. The velocity is not less than $7 \mathrm{~m} / \mathrm{s}$. This technology is the key for achieving high-speed operation and the production of all steel. 
In the secondary cooling zone, an online measurement method for secondary cooling water and special devices for the caster with the chamfered mold were developed in order to solve the problem of monitoring deficiencies in secondary water, water leakage in the equipment and an abnormal operating state for the nozzle. In accordance with the nozzle arrangement, many special devices fixed into the space of the dummy bar enter the secondary cooling zone together with the dummy bar. After casting, when the dummy bar moves down, the devices collect water volume of the secondary cooling zone. Then, the cooling state is judged by an artificial reading. It can reflect the actual cooling condition of chamfered slabs in the shortest possible time. It is greatly significant for the actual production of continuous casting slabs.

\subsection{The rapid adjustment technology of the roll gap and the arc}

The main accuracy factors affecting the quality of chamfered slabs include the roll gap of the segment and the out arc of the caster. For the application of chamfered mold technology, the adjustment technology of the accuracy for the equipment of the caster was improved. The adjustment technologies of the roll gap and the out arc were developed. They ensure the stable, efficient production of chamfered slabs.

The adjustment technologies of the roll gap are described as follows. According to the structural characteristics of the segment, a system of equations for the roll gap adjustment is obtained by an analytical method. Before adjustment, the roll gap in the import and export of the segment was measured. The difference from the standard roll gap was calculated. The number of the gaskets in the import and export segment is obtained by the difference and the system of equations. The roll gap of the segments can be adjusted properly. It took an hour at the most.

The adjustment technologies of the out arc are described as follows. According to the structural characteristics of the segment and caster, using CAD simulation, a system of equations for rapid adjustment is obtained. A mathematical model is established to obtain a function form. The amount of adjustment is directly obtained using this form and the measured values. The out arc of the segments can be adjusted properly. The precision can be guaranteed within $0.3 \mathrm{~mm}$.

\section{Results and discussion}

The formation mechanism of surface crack defects on micro-alloy steel during continuous casting was studied by investigation of corner transverse cracks, rolled steel defects, literature research, numerical simulation, field tests, online testing of the slab temperature and thermal simulation in the lab. A mathematical model of the steel flow, heat transfer, and macroscopic solidification within the right-angled and chamfered molds was established to analyze the effect of changes in the length of the chamfer, angle of the chamfer and corner shape to flow on the slab corner, temperature, solidification and straightening process.

Large chamfer mold technology was developed through the study of corner transverse cracks on continuous casting slabs. The weakening of two-dimensional cooling of corners, raising the slab temperature of the corners, improvement of the stress state, and reducing bending of the slab and straightening strain were obtained by changing the right angle to an obtuse angle larger than $90^{\circ}$ to eliminate and control the occurrence of corner transverse cracks on slabs and edge straight-line cracks on the strip eventually.

\subsection{Chamfer angle and chamfer length of chamfered mold theory}

The simulation molds are shown in Figure 10 , which are $1 / 4$ molds, due to the symmetry of the molds. The casting speed is $1.0 \mathrm{~m} / \mathrm{min}$. The length of the mold is $900 \mathrm{~mm}$. The width of the slab is $2000 \mathrm{~mm}$, and the thickness is $261 \mathrm{~mm}$. The temperature of the molten steel is $1535^{\circ}$, and the superheat is $25^{\circ}$. The chamfered angles $\alpha$ are $0^{\circ}, 22^{\circ}$, $30^{\circ}$ and $45^{\circ}$, and the lengths of the chamfered face are $10 \mathrm{~mm}, 40 \mathrm{~mm}, 60 \mathrm{~mm}$ and 

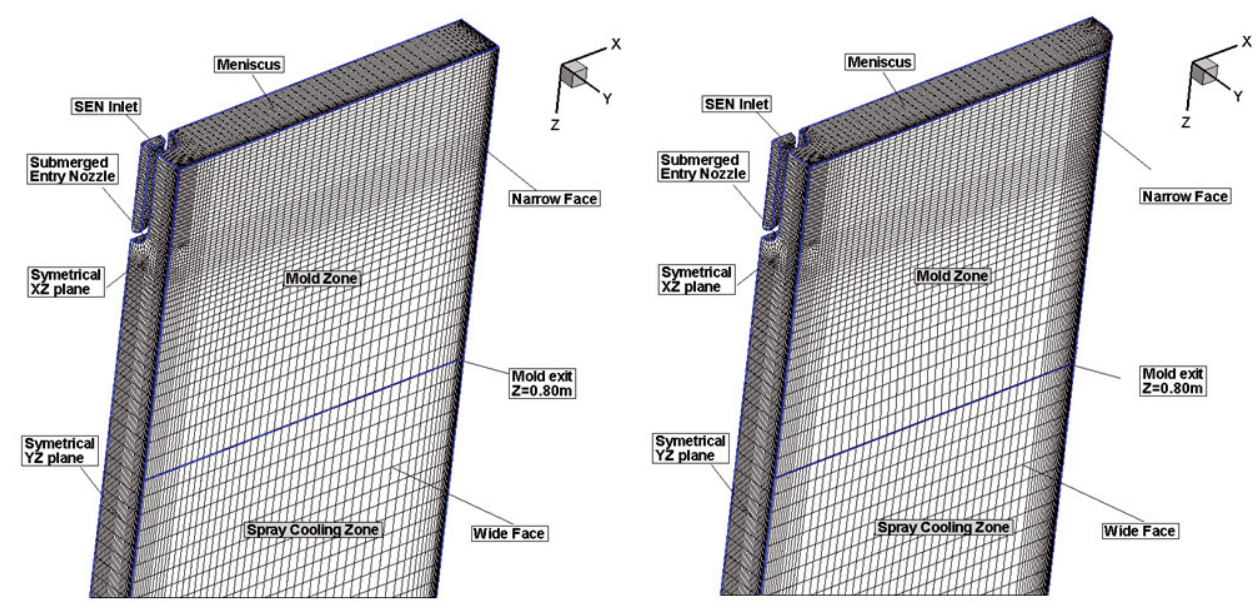

Fig. 10. The symmetrical geometric models of the rectangular and chamfered molds.

(a)

(b)
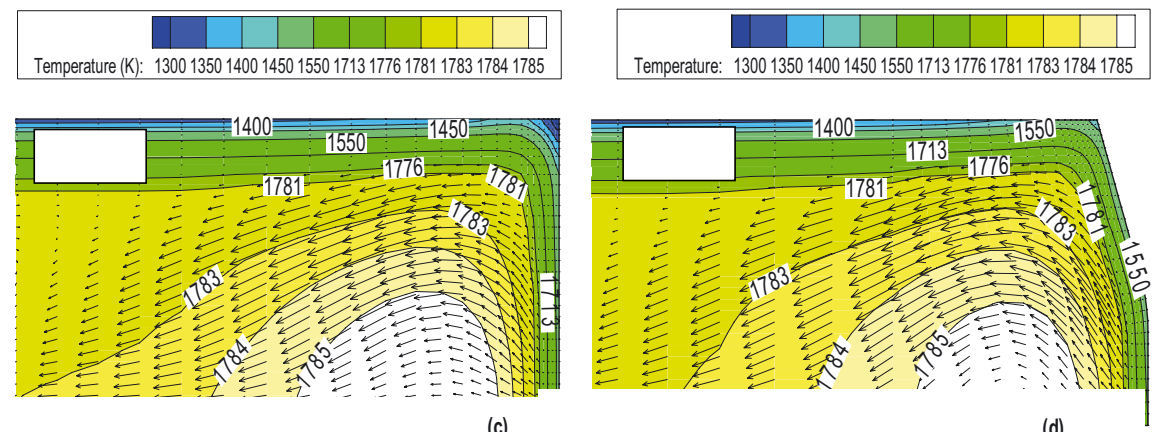

(c)
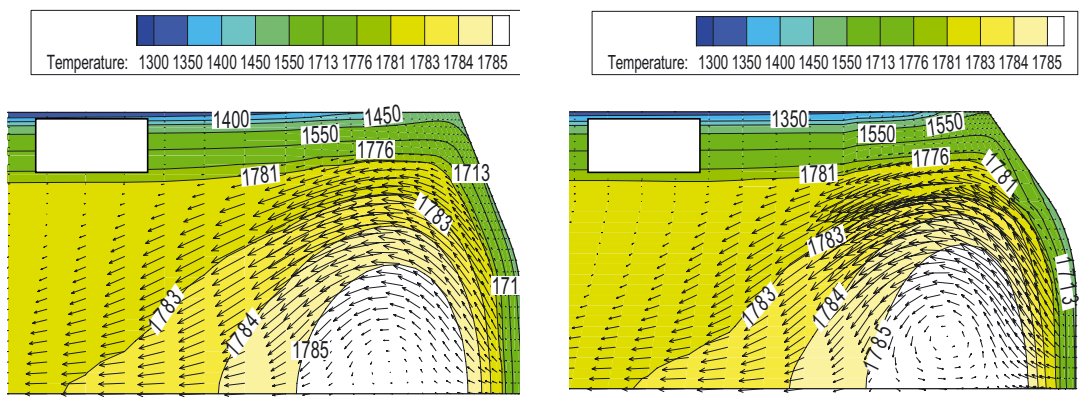

Fig. 11. The theoretical results of the chamfered mold's chamfer angle.

$80 \mathrm{~mm}$. The average heat flows of the narrow face and wide face are $1.28 \mathrm{MW} \cdot \mathrm{m}^{-2} \cdot \mathrm{k}^{-1}$ and $1.35 \mathrm{MW} \cdot \mathrm{m}^{-2} \cdot \mathrm{k}^{-1}$.

As shown in the following, the simulation results show that the temperature is increased near the chamfered corner, and the temperature increases with the enhancement of the corner $\alpha$. The best angle size is $30^{\circ}$ of the narrow copper of the chamfered mold, regarding the liquid steel flow on the mold's corner, surface horizontal velocity of the liquid steel, temperature distribution on the mold's corner of the outlet slab and the shell thickness of slab corners.

The research shows that the best chamfer length is $40 \sim 60 \mathrm{~mm}$, which is the effect of the chamfer length to meniscus velocity field, which can be seen in Figure 12. With the increase in the chamfer length, the fluid flow near the intersection of the wide side and narrow side and its impingement on the slab corner are stronger. It also affects the 


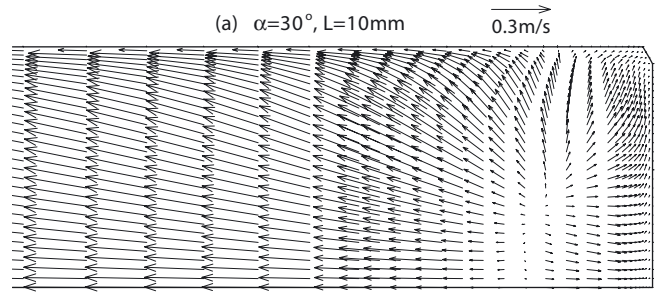

(c) $\alpha=30^{\circ}, \mathrm{L}=60 \mathrm{~mm}$

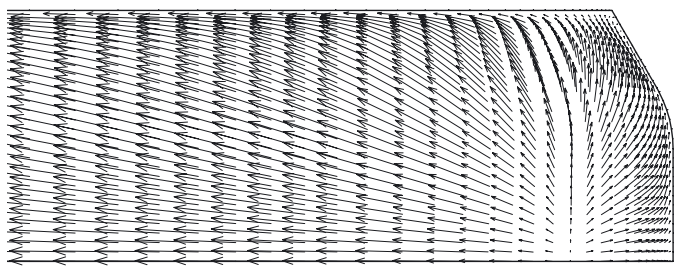

(b) $\alpha=30^{\circ}, \mathrm{L}=40 \mathrm{~mm}$

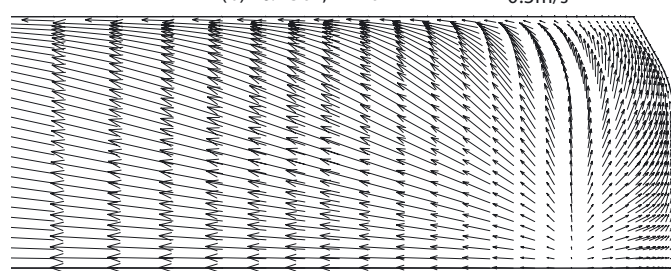

(d) $\alpha=30^{\circ}, \mathrm{L}=80 \mathrm{~mm} \quad \overrightarrow{0.3 \mathrm{~m} / \mathrm{s}}$

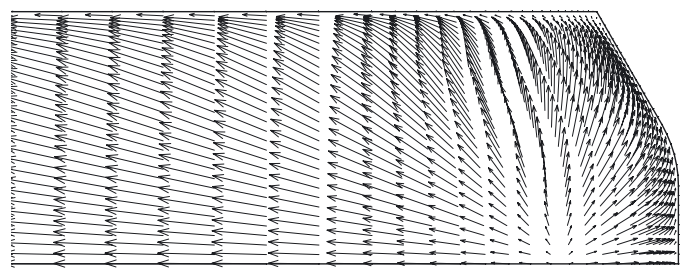

Fig. 12. The theoretical results of the chamfered mold's chamfer length.
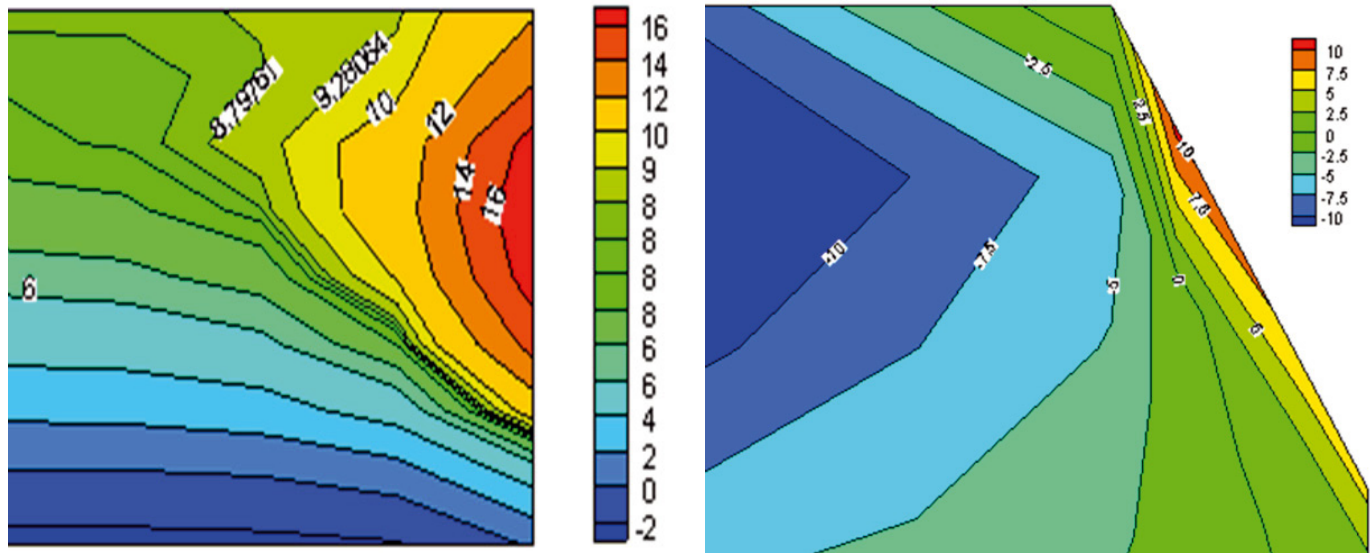

Fig. 13. Tensile stress in the casting direction during the straightening process of slabs.

formation of the primary solidifying shell at the slab corner. So, the chamfer mold should be designed with a suitable length.

\subsection{Study on stress near the corner during the straightening process}

The tensile stress in the casting direction is the main reason for transverse cracks $[9,10]$. Reducing the tensile stress of the slab corner can reduce the risk of corner cracks effectively. Comparing the simulation results, the maximum stress value of the edge from a rectangular slab, $16 \mathrm{MPa}$, is reduced in a chamfered slab $10 \mathrm{MPa}$. The chamfered slab reduces the tensile stress at the same straightening conduction, and the maximum stress is reduced by $37.5 \%$ compared with the rectangular slabs.

$30^{\circ}$ was the optimal angle of the narrow chamfer. $L=40-60 \mathrm{~mm}$ was the optimal length of the chamfer. The angle was $30^{\circ}$ in the steelworks of Hanbao, while the length was $57 \mathrm{~mm}$. the chamfered slab which is produced by the chamfered mold can be seen in Figure 14.

\subsection{The effect of the chamfered mold}

This project was applied to developing a large chamfer narrow face copper plate and 


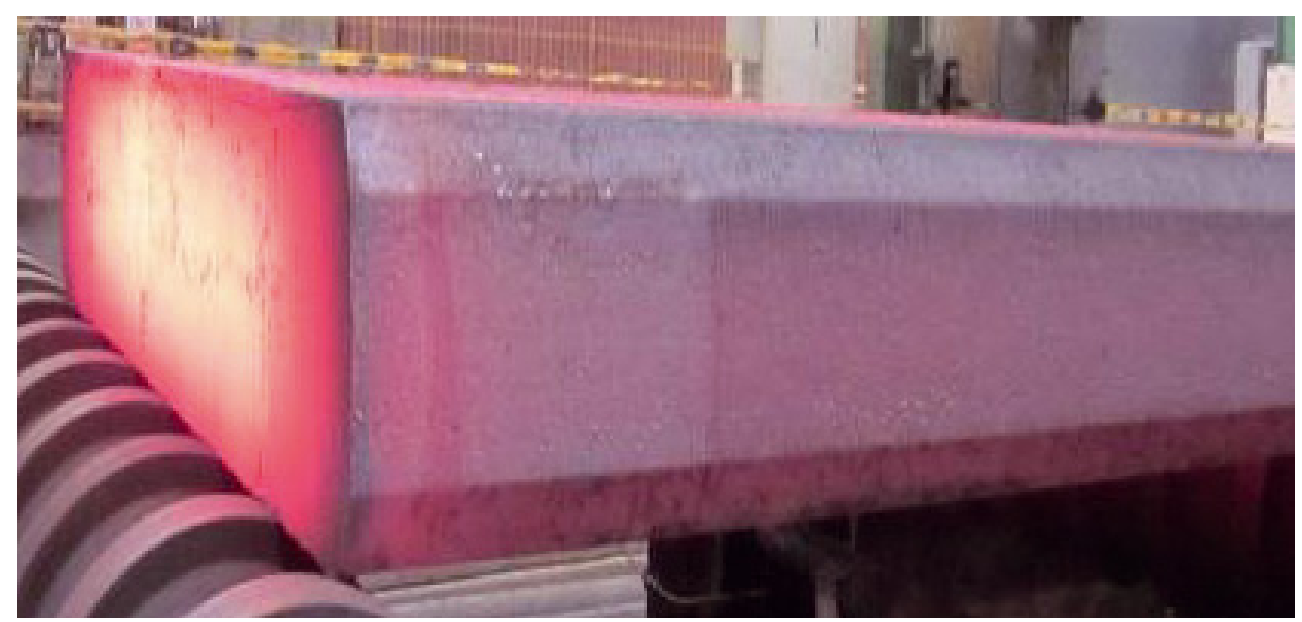

Fig. 14. The produced chamfered slab.

supporting equipment and technology systems. For a typical micro-alloyed steel slab in large slab caster production, the targets achieved are shown as follows:

(1) The slab was produced cumulatively at 354.248 ten thousand tons in a 1031 furnace from 2013.1 to 2013.12. The slabs produced slabs were the same as the slabs produced by the original caster. The incidence of corner cracks was nearly 0 . The slabs were hot-charged to rolling without corner cuts. The defect rate of rolled steel on the edge was $<4 \%$.

(2) The suitable cast speed of micro-alloyed steel was $1.0 \sim 1.2 \mathrm{~m} / \mathrm{min}$, while that of low-carbon steel was $1.35 \mathrm{~m} / \mathrm{min}$. This speed range is a normal cast speed for mainstream slab casters on the worldwide.

(3) The use life of the chamfered mold was improved greatly, as was the innovation of assembly and maintenance technology. The average use lifetime of narrow side copper $>8$ ten thousand ton. The annual average normal lifetime was 10.25 ten thousand ton, while the longest lifetime reached 16.37 ten thousand ton, exceeding the use lifetime of traditional right-angled copper plate. This use lifetime was higher than the others all over the world.

(4) The chamfered mold has been employed steadily in industrial production, and was used on the two-part four-flow casters of Hanbao. Chamfered slab was produced to 354.25 ten thousand ton, and the monthly maximum was 46.3 ten thousand ton. Micro-alloyed steel production reached 76.29 ten thousand tons in the whole year, a total reduction of 1014 tons of corners cut (by $0.133 \%$ proportion).

(5) There were 60 ten thousand ton micro alloyed steel hot-charged in 2013. Compared with the hot charging, coal consumption per ton of steel was $32.6 \mathrm{~kg}$ with the cold charging. From the viewpoint of greenhouse gas emissions, $1 \mathrm{~kg}$ per ton of coal combustion produces $2.7 \mathrm{~kg} \mathrm{CO}$, while the incremental emission per ton of steel was $81 \mathrm{~kg} / \mathrm{t}$. $\mathrm{CO}_{2}$ emissions could be reduced by 48600 tons/year by hot charging in one full year.

\section{Conclusions}

- The tapered design and management practices were produced base on the research of the taper variation of the narrow side of the mold during the casting process. Technological bottlenecks of corner longitudinal cracks and corner longitudinal breakouts were broken. The technology has been applied in largescale industrial production, compared with other steel mills.

- The highest excessive steel production amount was over 163700 tons by the chamfered mold used in Hanbao Steel 
mills, which was the highest on record from invention to industrial applications, while the excessive steel amount was less than 4.5 ten thousand ton in other steel mills so far.

- Corner transverse cracks of microalloyed steel slabs were solved fundamentally by this project, and it is significant in the promotion of efficient and low-cost production of niobium, vanadium and titanium micro-alloyed steel.

\section{Acknowledgements}

The authors wish to thank the National Natural Science Foundation of China, for their financial supports to key Project No. 51204059.

\section{References}

[1] Y.M. Won, H.N. Han, T.J. Yeo, ISIJ Int. 40 (2000) 129-136

[2] S. Harade, S. Tanaka, H. Misumi, ISIJ Int. 30 (1990) 310-316

[3] B.G. Thomas, ISIJ Int. 35 (1995) 6-10

[4] H.N. Han, J.E. Lee, T.J. Yeo, ISIJ Int. 39 (1999) $445-454$

[5] R. Saraswat, D.M. Maijer, P.D. Lee, ISIJ Int. 47 (2007) 95-104

[6] Y. Gan, C.Z. Yang, H. Zhang, Patent, China, 102274934A, 2011-12-14

[7] H. Zhang, M. Wang, Y. M. Wu, Patent, China, 202146982U, 2012-02-22

[8] M.L. Wang, H. Zhang, H.B. Tao, Patent, China, 202239532U, 2012-05030

[9] S.J. Lee, J.W. Choi, Metall. Res. Technol. 3 (2008) 127-134

[10] P. Hu, H. Zhang, ISIJ Int. 54 (2014) 2283-2287 